\title{
Synthesis and Spectroscopic Characterization of Copper(II)-Nitrito Complexes with Hydrotris(pyrazolyl)borate and related Coligands
}

\author{
Nicolai Lehnert ${ }^{* 1}$, Ursula Cornelissen ${ }^{2}$, Frank Neese* ${ }^{3}$, Tetsuya Ono ${ }^{4}$, Yuki \\ Noguchi ${ }^{4}$, Ken-ichi Okamoto ${ }^{4}$, Kiyoshi Fujisawa* ${ }^{4}$ \\ Department of Chemistry, The University of Michigan, 930 N. University, Ann Arbor, MI_48109, USA \\ Institut für Anorganische Chemie, Christian-Albrechts-Universität Kiel, Olshausenstrasse 40, D-24098 \\ Kiel, Germany \\ Institut für Physikalische and Theoretische Chemie, Rheinische Friedrich-Wilhelms-Universität Bonn, \\ Wegelerstrasse 12, 53115 Bonn, Germany \\ Graduate School of Pure and Applied Sciences, University of Tsukuba, Department of Chemistry, \\ University of Tsukuba, Tsukuba 305-8571, Japan
}

\section{Supporting Information}

\footnotetext{
| ${ }^{1}$ The University of Michigan, Ann Arbor, MI_48109, USA

${ }^{2}$ Christian-Albrechts-Universität Kiel, D-24098 Kiel, Germany

${ }^{3}$ Rheinische Friedrich-Wilhelms-Universität Bonn, 53115 Bonn, Germany

${ }^{4}$ University of Tsukuba, Tsukuba 305-8571, Japan
} 


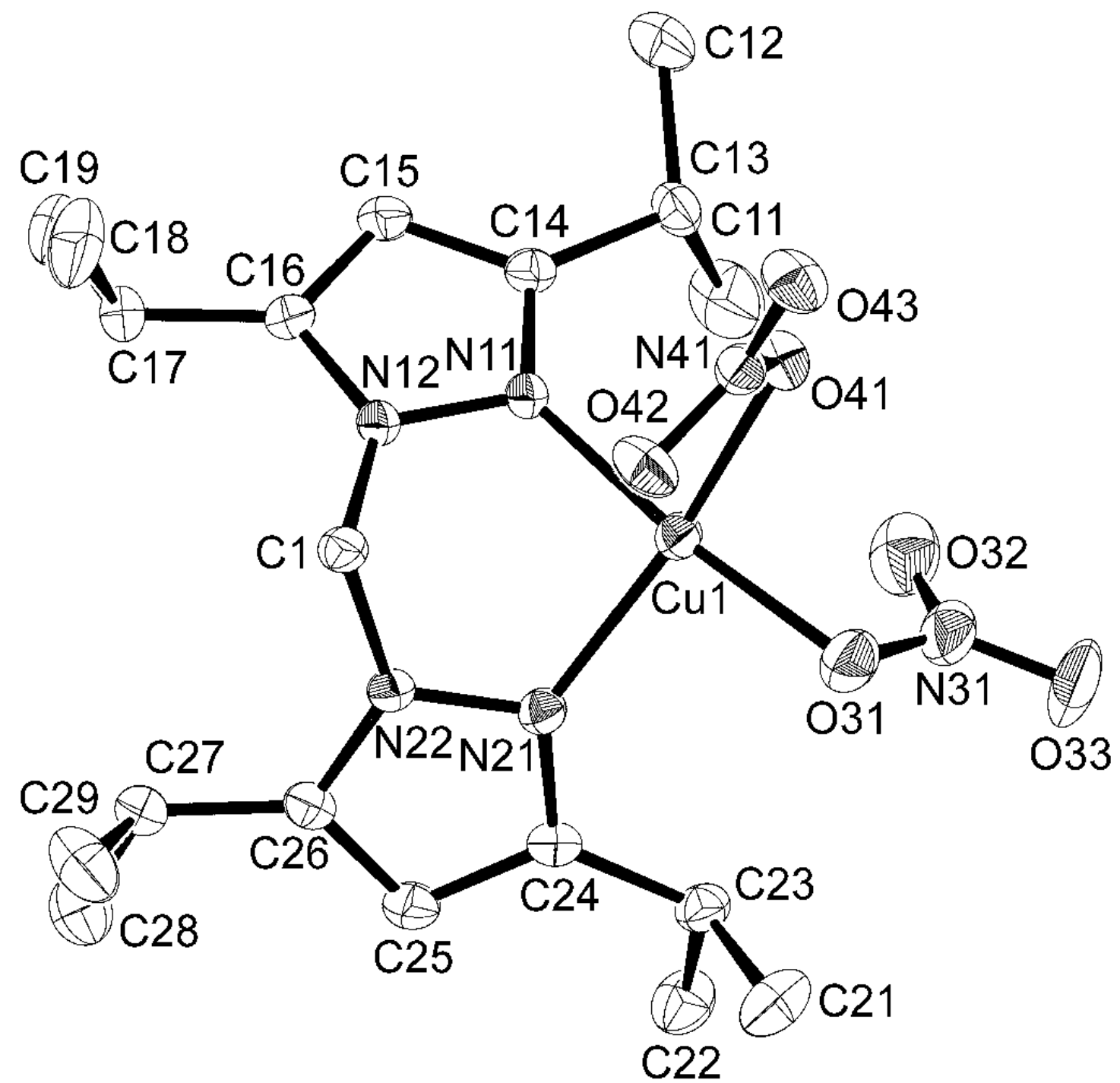

Figure S1. Crystal structure of $\left[\mathrm{Cu}\left(\mathrm{L} 1{ }^{\prime \prime}\right)\left(\eta^{1}-\mathrm{NO}_{3}\right)_{2}\right]$ (5) (50\% probability ellipsoids shown). 


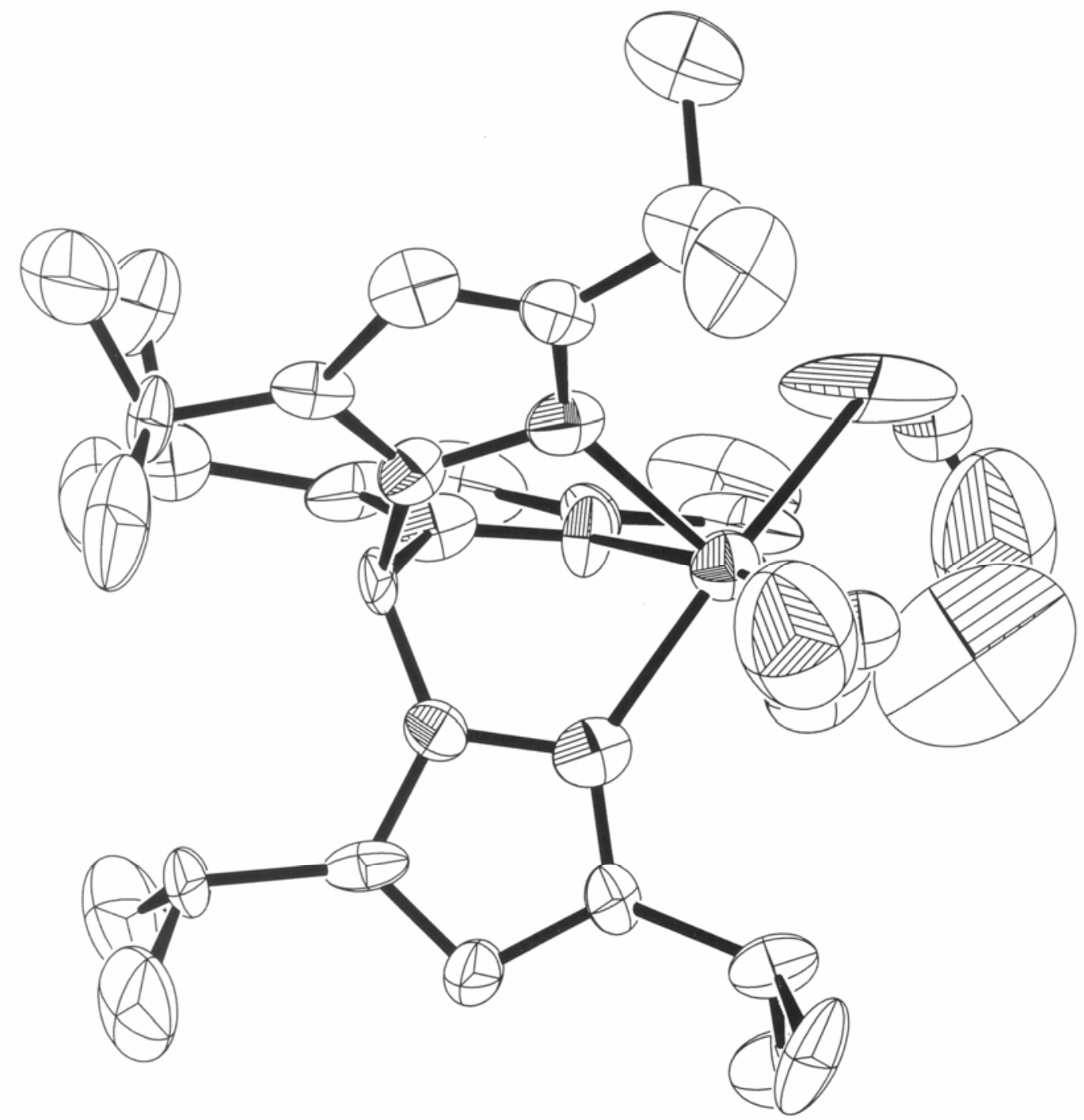

Figure S2. Preliminary crystal structure of $\left[\mathrm{Cu}\left(\mathrm{L} 1^{\prime}\right)\left(\eta^{1}-\mathrm{ONO}\right)\left(\eta^{1}-\mathrm{NO}_{2}\right)\right](3)(50 \%$ probability ellipsoids shown). The nitrite ligands are somewhat disordered in this data set, but the structure strongly indicates that the two coordinated $\mathrm{NO}_{2}{ }^{-}$ligands have a different coordination mode in this complex. This is in agreement with the available spectroscopic data. 


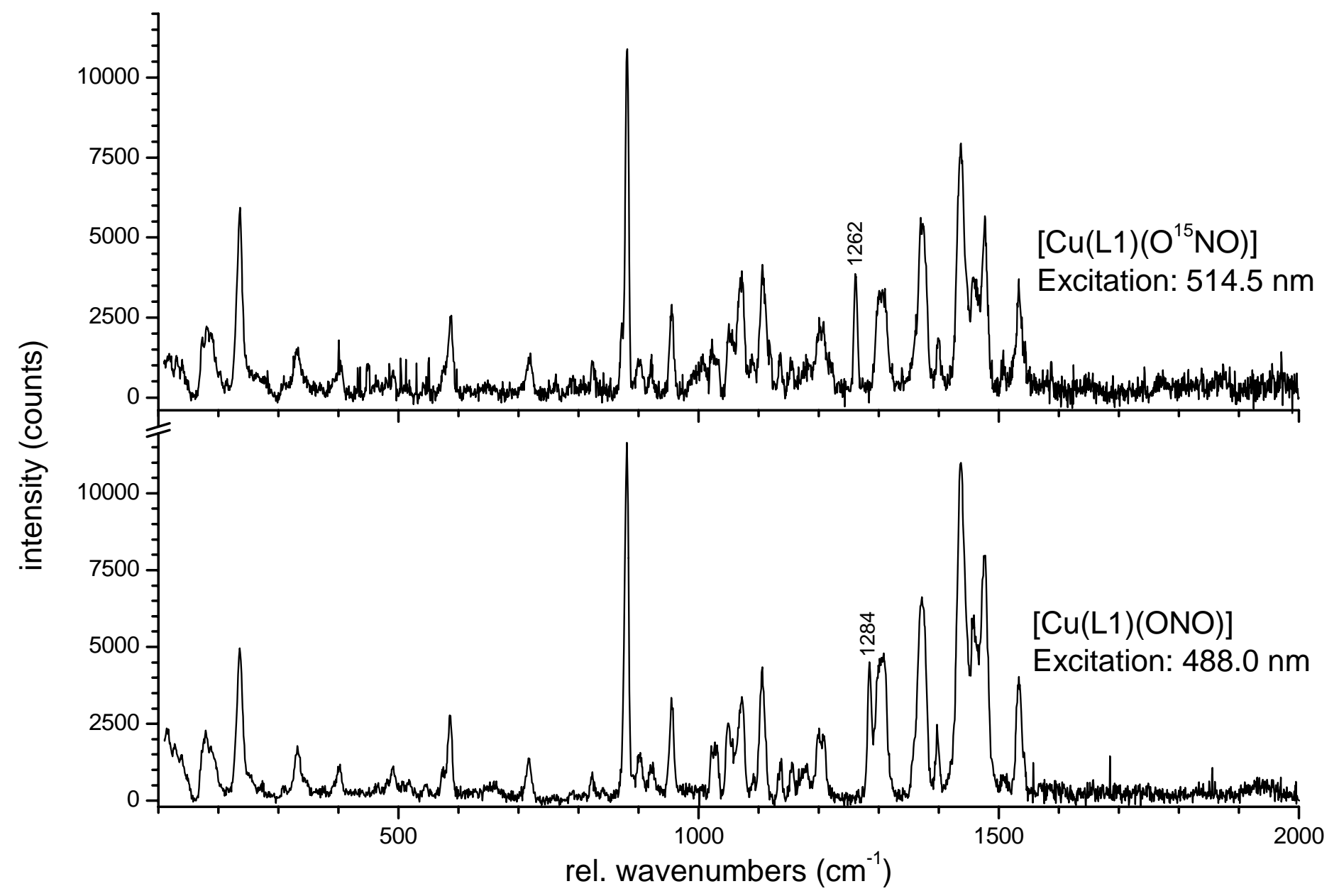

Figure S3. Raman spectrum of $\mathbf{1}$ (bottom) and of the corresponding ${ }^{15} \mathrm{NO}_{2}$ isotope labeled complex (top). 

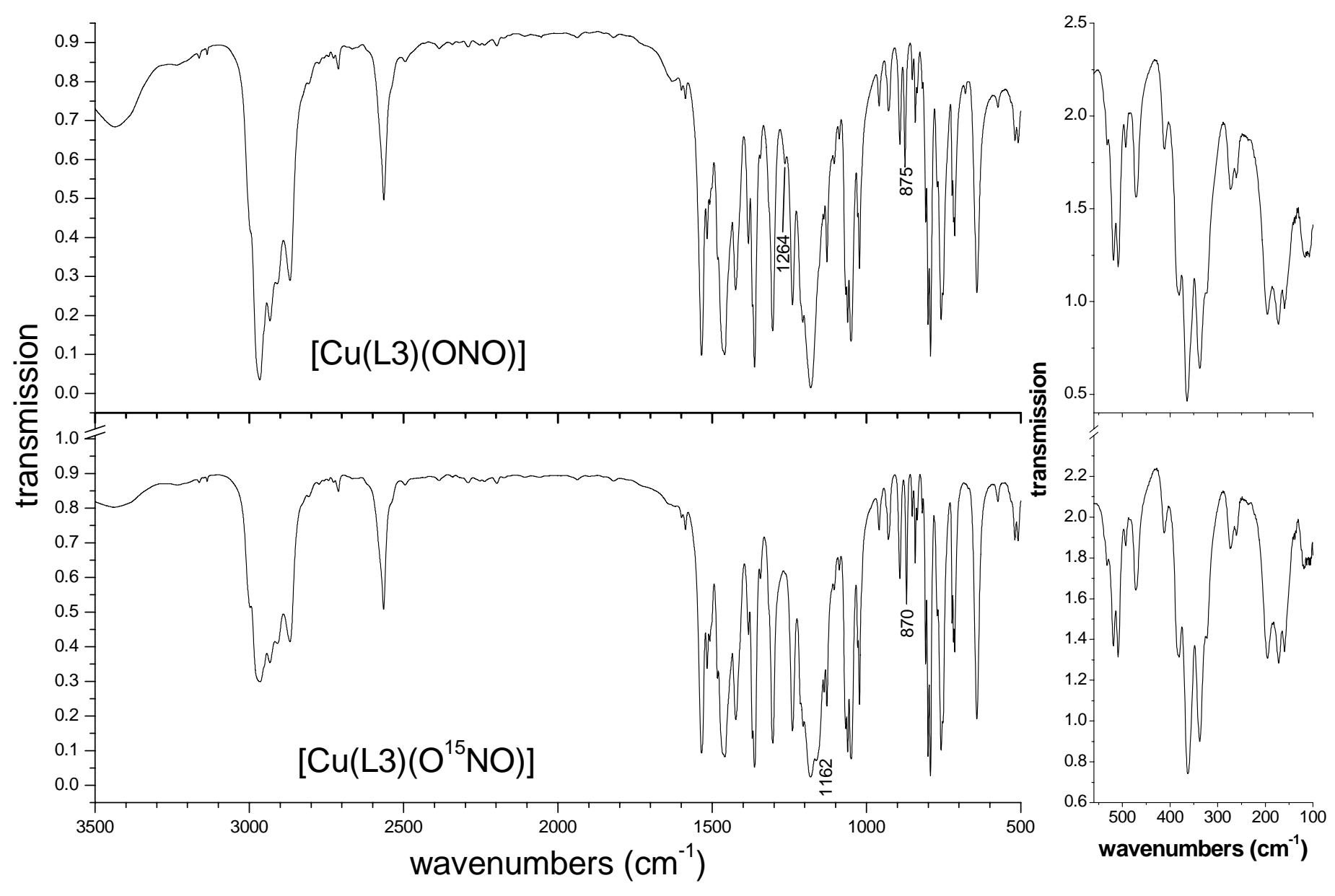

Figure S4. IR spectrum of 2 (top) and of the corresponding ${ }^{15} \mathrm{NO}_{2}$ isotope labeled complex (bottom). 


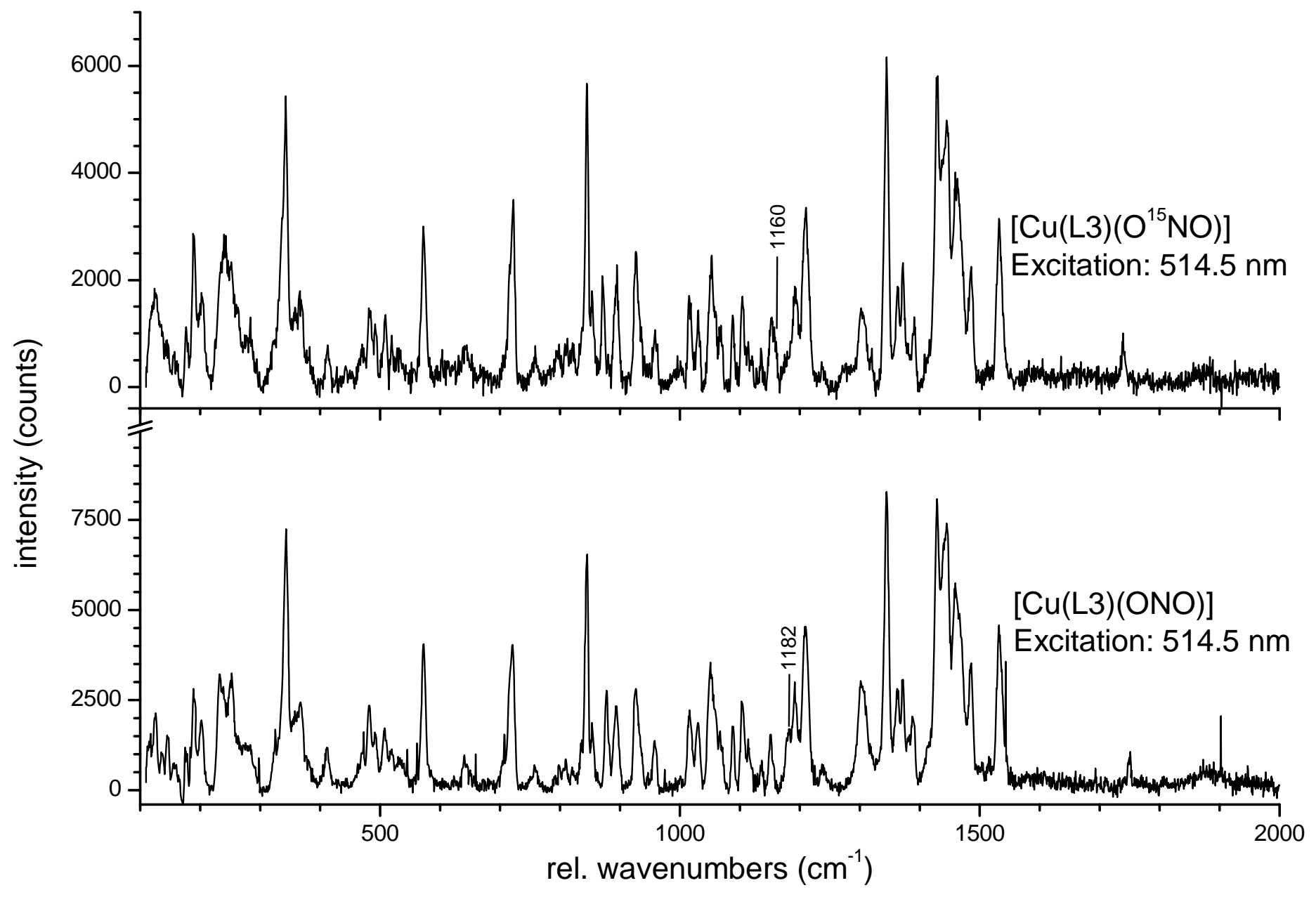

Figure S5. Raman spectrum of $\mathbf{2}$ (bottom) and of the corresponding ${ }^{15} \mathrm{NO}_{2}$ isotope labeled complex (top). 

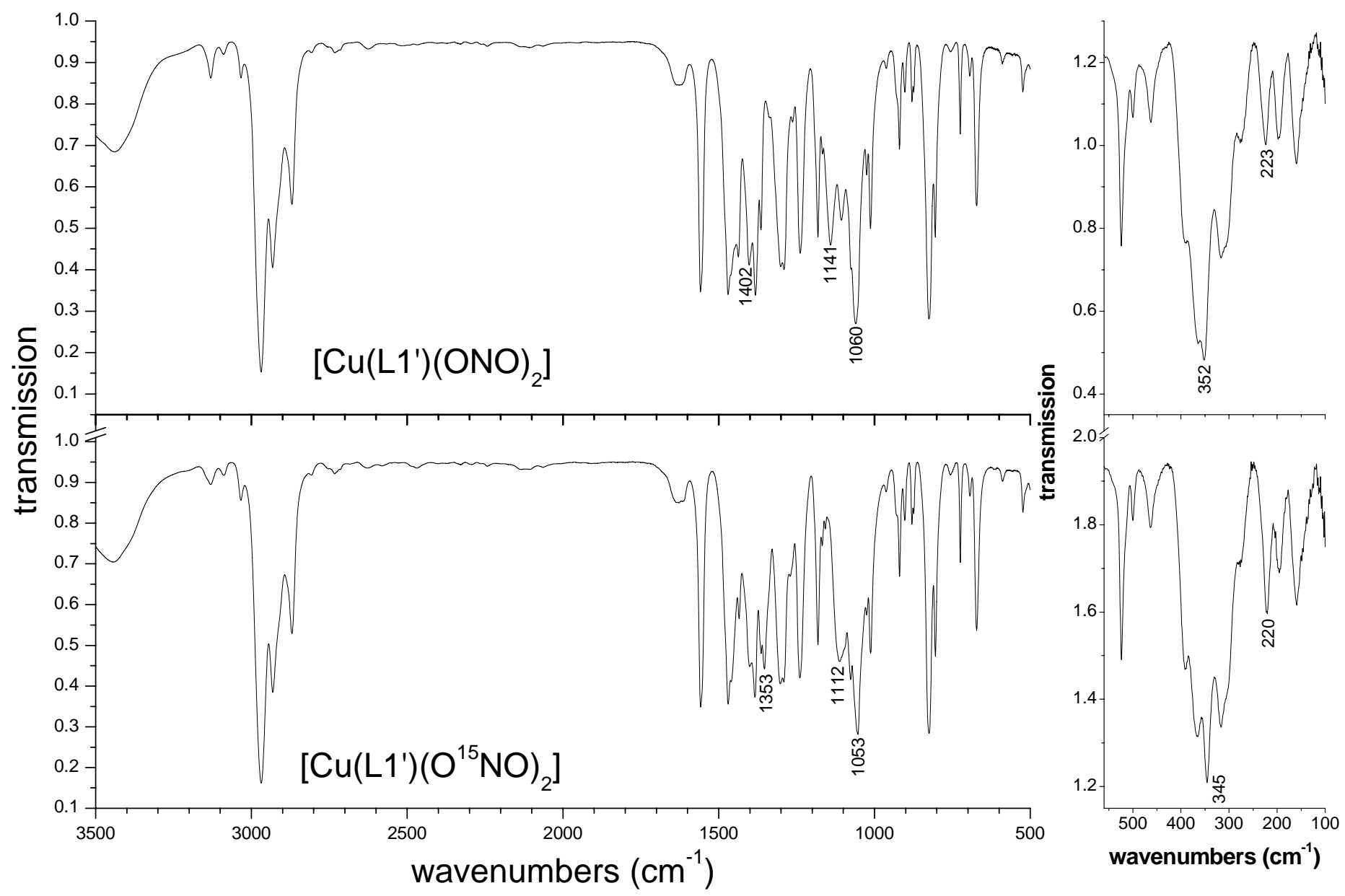

Figure S6. IR spectrum of 3 (top) and of the corresponding ${ }^{15} \mathrm{NO}_{2}$ isotope labeled complex (bottom). 


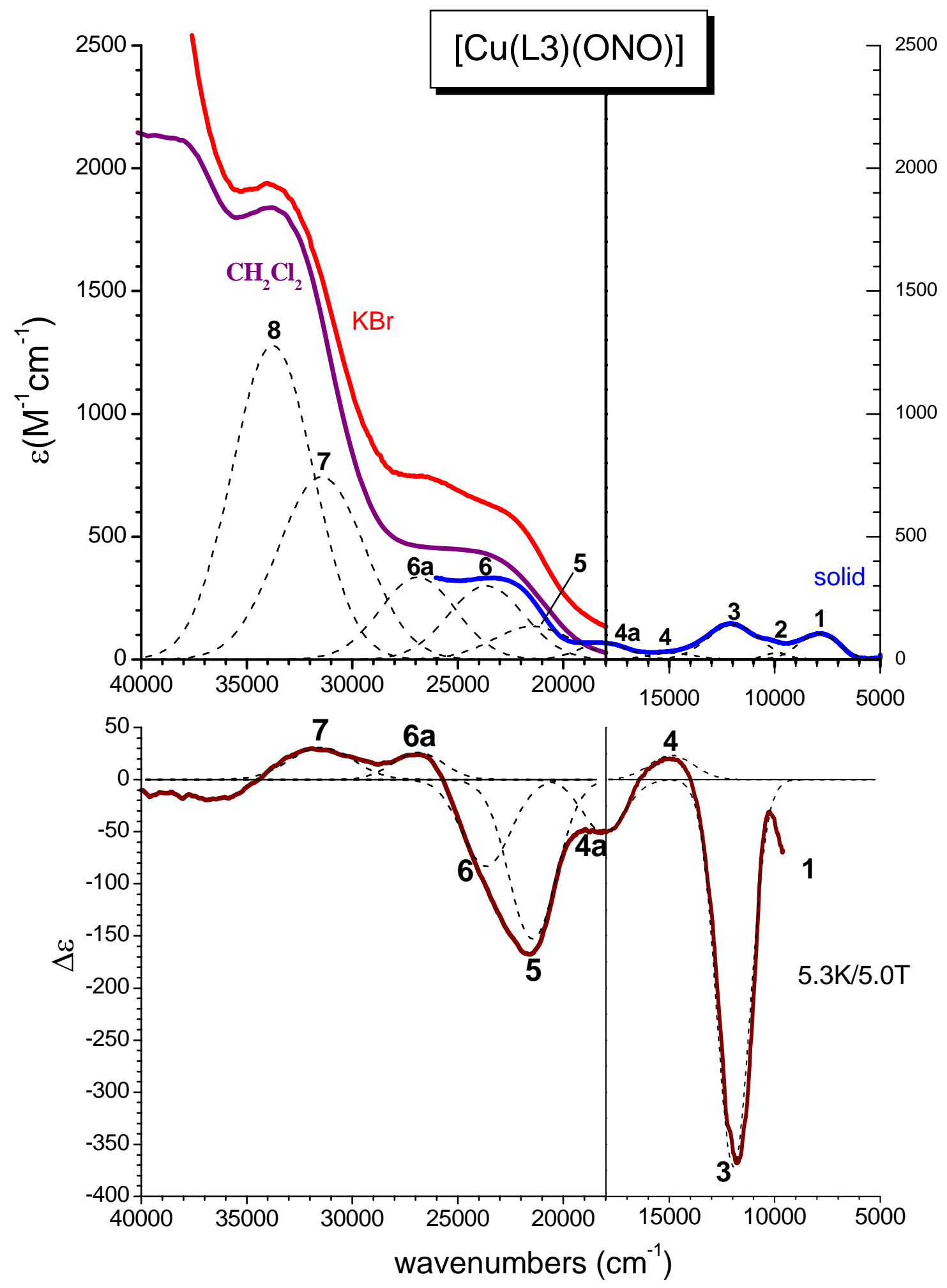

Figure S7. UV-Vis absorption (top) and MCD spectrum (bottom) of 2. The absorption spectrum in the UV-Vis region was recorded in $\mathrm{CH}_{2} \mathrm{Cl}_{2}$ at low temperature; in the NIR region, the material was pressed between sapphire windows and recorded at $20 \mathrm{~K}$ (see Experimental Section). The MCD spectrum was obtained on mulls. Also included are Gaussians obtained from a simultaneous fit of these data. The absorption spectrum recorded in a $\mathrm{KBr}$ disk is also shown as indicated. 


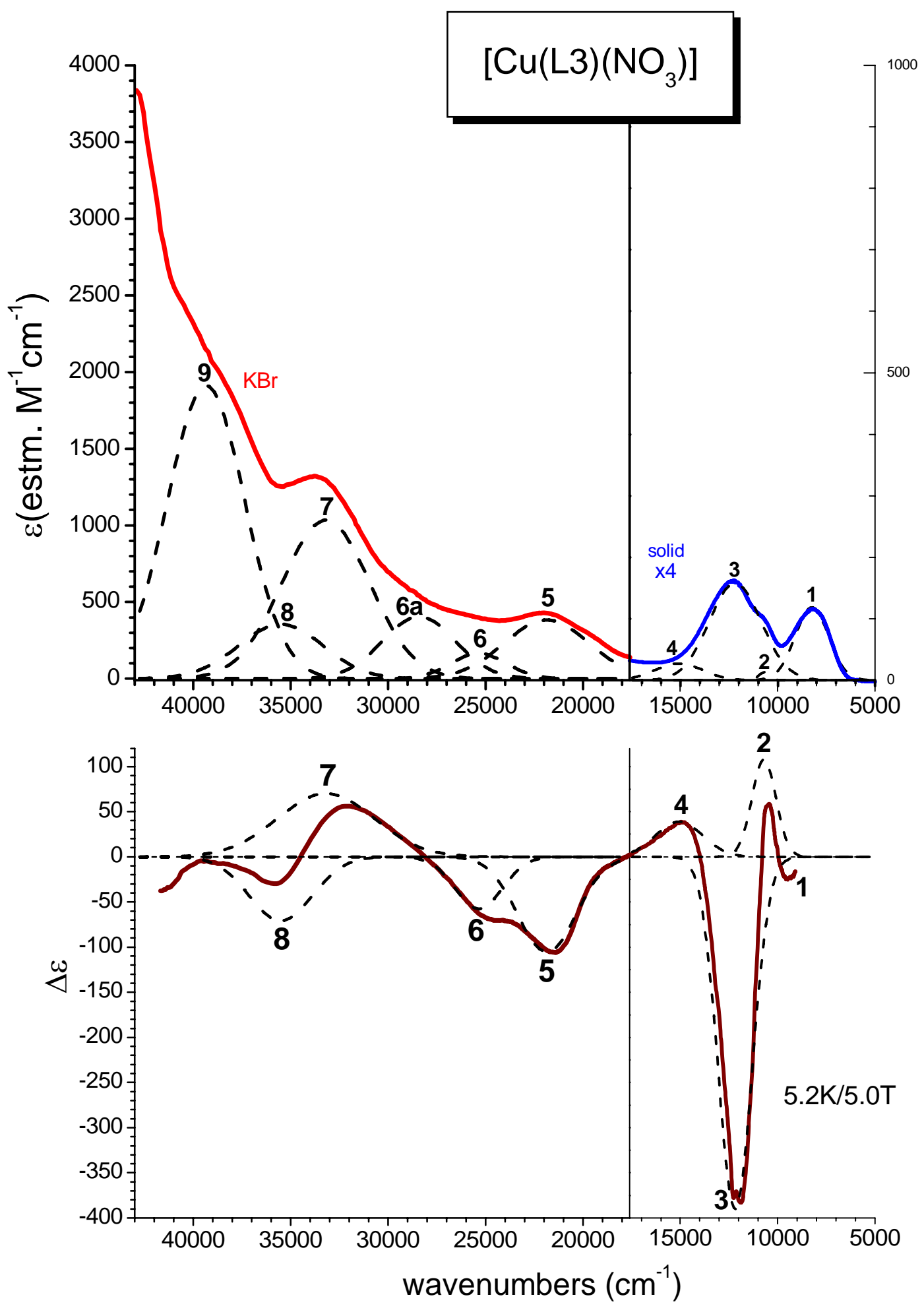

Figure S8. UV-Vis absorption (top) and MCD spectrum (bottom) of [Cu(L3)(NO $\left.\left.\mathrm{NO}_{3}\right)\right]$. The absorption spectrum in the UV-Vis region was recorded in a $\mathrm{KBr}$ disc at $20 \mathrm{~K}$; in the NIR region, the material was pressed between sapphire windows and recorded at $20 \mathrm{~K}$ (see Experimental Section). The MCD spectrum was obtained on mulls. Also included are Gaussians obtained from a simultaneous fit of these data. 


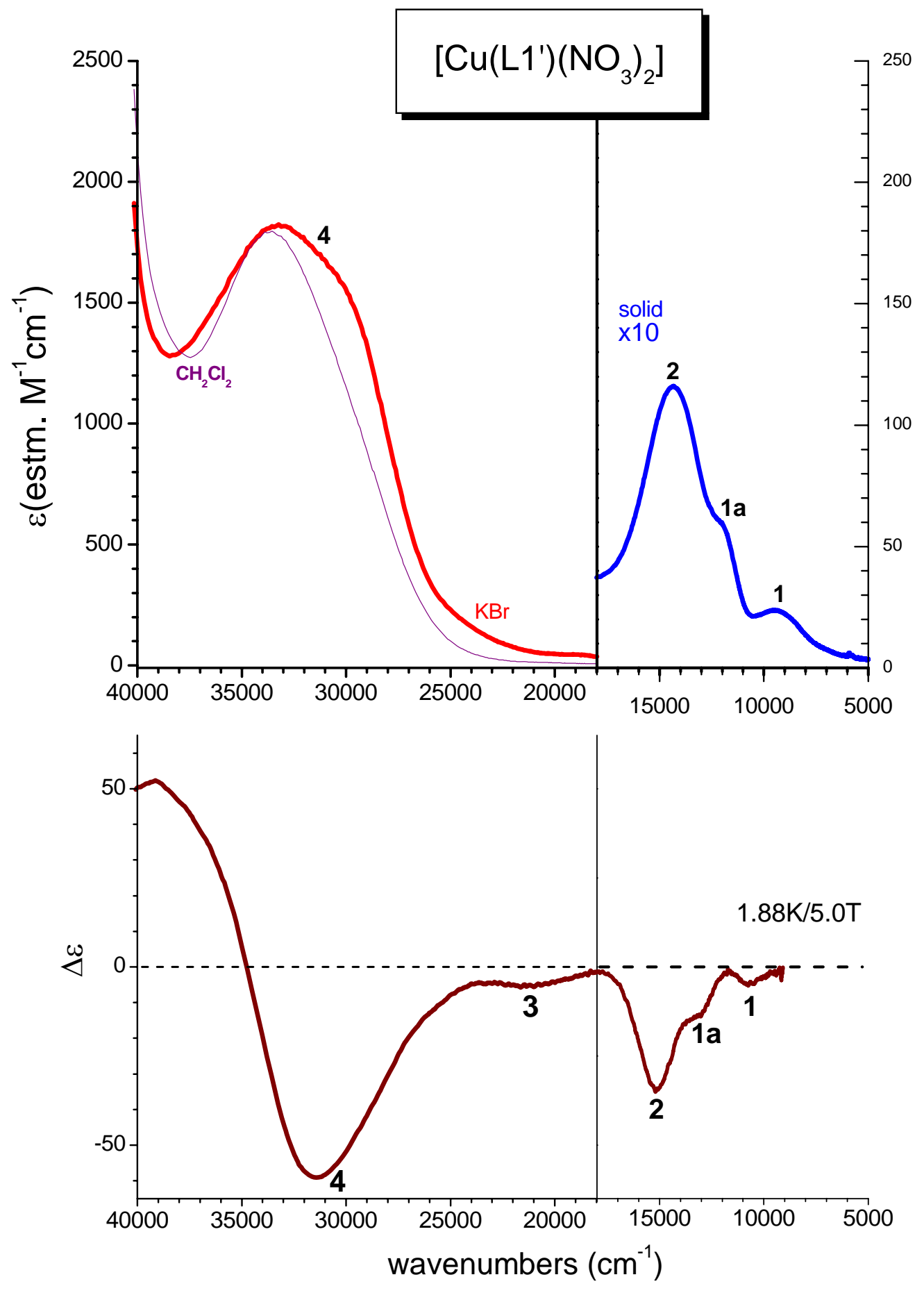

Figure S9. UV-Vis absorption (top) and MCD spectrum (bottom) of [Cu(L1') $\left.\left(\mathrm{NO}_{3}\right)_{2}\right]$. The absorption spectrum in the UV-Vis region was recorded in a $\mathrm{KBr}$ disk at $20 \mathrm{~K}$; in the NIR region, the material was pressed between sapphire windows and recorded at $20 \mathrm{~K}$ (see Experimental Section). The MCD spectrum was obtained on mulls. The absorption spectrum recorded in solution is also shown as indicated. Note that a simultaneous fit of the absorption and MCD data was not possible. This is due to the fact that the absorption and MCD bands do not line up; although their overall appearance is similar. The reason for this is not clear (see text). 


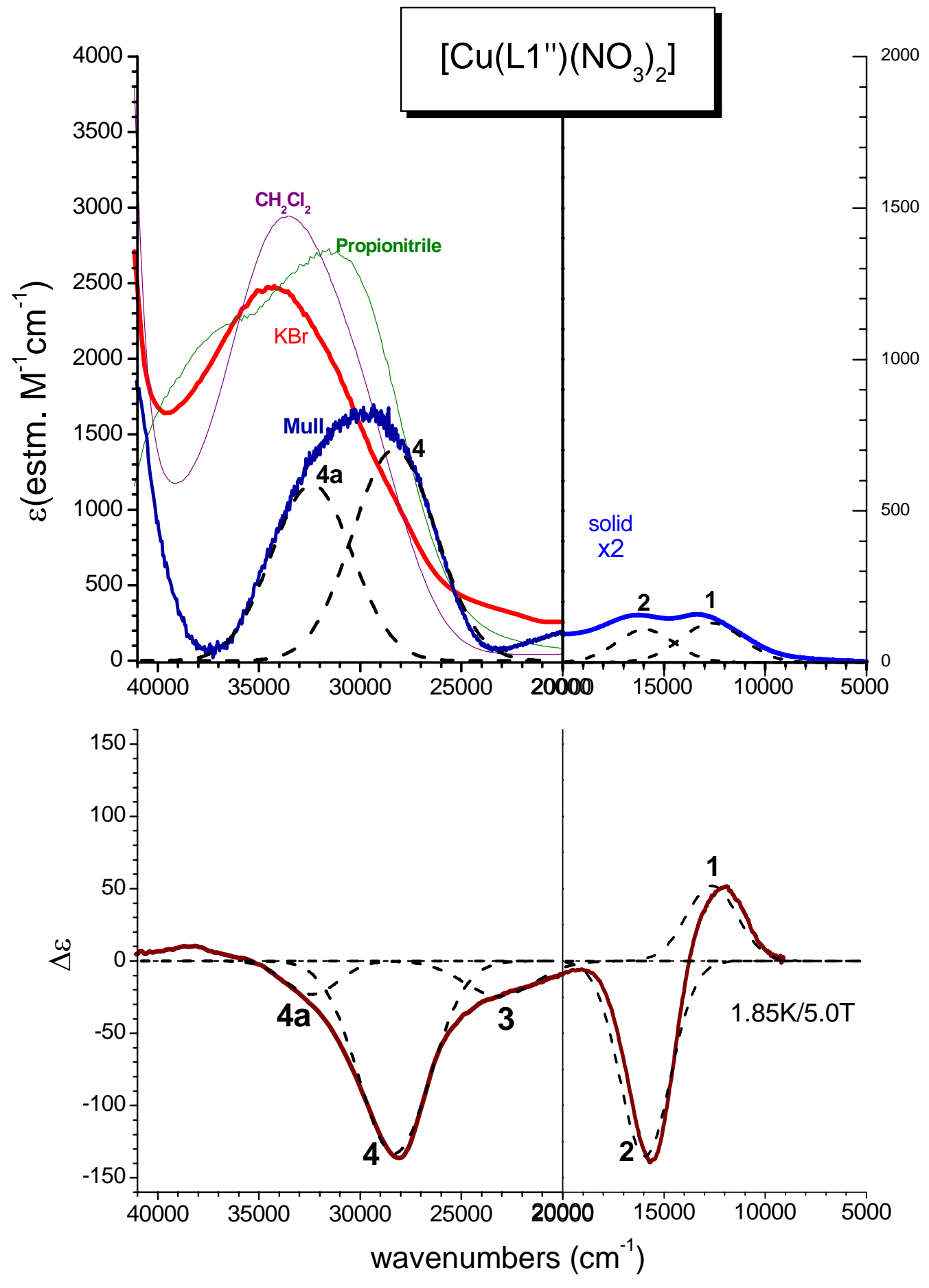

Figure S10. UV-Vis absorption (top) and MCD spectrum (bottom) of [Cu(L1' ') $\left(\mathrm{NO}_{3}\right)_{2}$ ] (5). The absorption spectrum in the UV-Vis region was recorded in a mull at 20K; in the NIR region, the material was pressed between sapphire windows and recorded at 20K (see Experimental Section). The MCD spectrum was obtained on mulls. Also included are Gaussians obtained from a simultaneous fit of these data. The absorption spectrum recorded in solution and in a $\mathrm{KBr}$ disk is also shown as indicated. Importantly, both the solution and the $\mathrm{KBr}$ data do not line up with the MCD and absorption data recorded in mulls. This shows that complex $\left[\mathrm{Cu}(\mathrm{L} 1\right.$ '” $\left.)\left(\mathrm{NO}_{3}\right)_{2}\right]$ must undergo some kind of isomerization in solution (see text). 


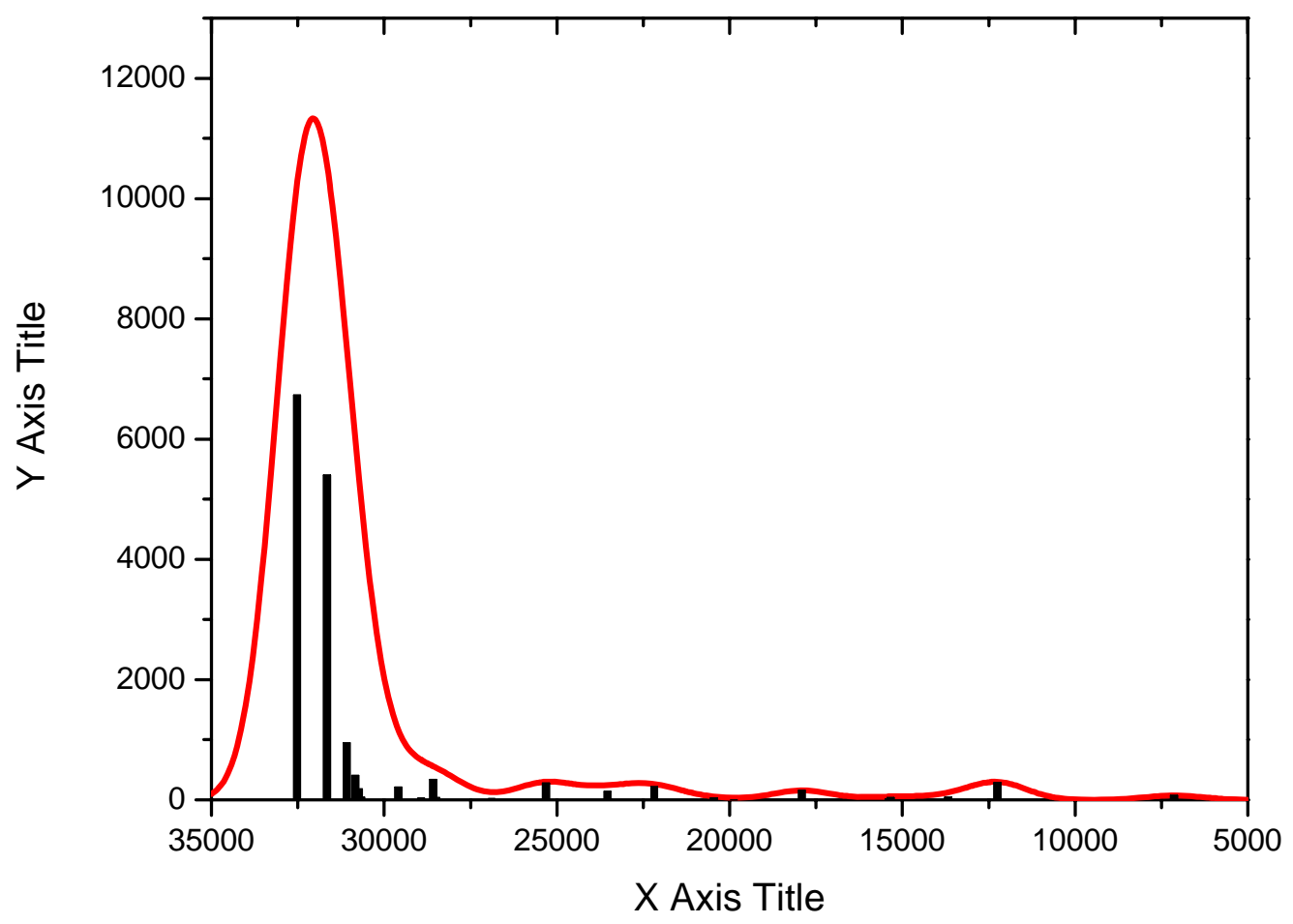

Figure S11. Calculated absorption spectrum of 1d. The intensity of the CT bands above $30000 \mathrm{~cm}^{-1}$ seems largely overestimated compared to experiment. 